\title{
Consciousness and the social brain (/review/10.5860/CHOICE.52-0554)
}

Graziano, Michael S. A. (/search?exactauthor=Graziano, Michael S. A) Oxford, 2013

268p, $9780199928644 \$ 29.95$

LC Call Number: BF (/search?lcstart=BF)311

Graziano (neuroscience, Princeton) presents a new theory of consciousness, one that argues that awareness--which he defines as a subset of consciousness--is an attention schema. The underlying assumption of the theory is that awareness is not simply passive watching; it serves to direct brain functioning. In the first half of the book Graziano lays out the theory; in the second half he relates the new theory to the many others already in existence and to contemporary research in neuroscience and other areas. He makes a clear and compelling case for the new approach, and he applies the theory to a number of interesting subjects, e.g., blindsight, mirror neurons, free will, and spirituality. He also presents a solid but brief summary of existing theories of consciousness. The book is clearly written but steeped in the language of neuroscience. As a result, readers without technical background and a strong interest in the discipline may find the book difficult to follow.

Summing Up: Recommended. Upper-division undergraduates and above.

Reviewer: R. Levine (/search?reviewer=R. Levine), California State University--Fresno Recommendation: Recommended Readership Level: Upper-division Undergraduates, Graduate Students, Researchers/Faculty Interdisciplinary Subjects:

Subject: Social \& Behavioral Sciences - Psychology (/search?q=\&sbj[]=psy)

Choice Issue: sep 2014 vol. 52 no. 1

Choice Review \#: 52-0554

Review DOI: 10.5860/CHOICE.52-0554 